\title{
PARTICIPACIÓN CIUDADANA Y CONTROL SOCIAL: LOS SERVICIOS PÚBLICOS DOMICILIARIOS EN BUCARAMANGA (COL.)
}

\author{
Eva del Pilar Plata Sarmiento
}

Doctorando en Derecho (c.) Universidad Externado de Colombia (Colombia); Especialista en Derecho Administrativo, Universidad Santo Tomás - Seccional Bucaramanga (Colombia); Abogada, Universidad Santo Tomás - Seccional Bucaramanga (Colombia). Docente - Investigadora Facultad de Derecho.

E-Mail: platasarmiento@gmail.com

\section{Resumen}

El texto presenta los resultados finales del proyecto de investigación Participación ciudadana en los Comités de Desarrollo y Control Social de las Comunas 5, 6 y 7 de Bucaramanga, cuyo estudio se centró en describir y caracterizar la relación existente entre participación ciudadana y funcionalidad de los Comités de Desarrollo y Control Social, a fin de determinar su incidencia en la gestión pública de los servicios públicos domiciliarios.

\section{Palabras Clave}

Servicio público, participación ciudadana, servicio público domiciliario, control social.

\begin{abstract}
The paper present the final results of the research Project Citizens participation in the Development and Social Control Committees in Communes 5, 6 and 7 of the city of Bucaramanga. The research was focused on describing and characterizing the existing relationship between citizen participation and functionality of the Development and social Control Committees, with the idea to determine its incidence in the public management of domiciliary public services.
\end{abstract}

\section{Key Words}

Public service, civic participation, domiciliary public services, social control 

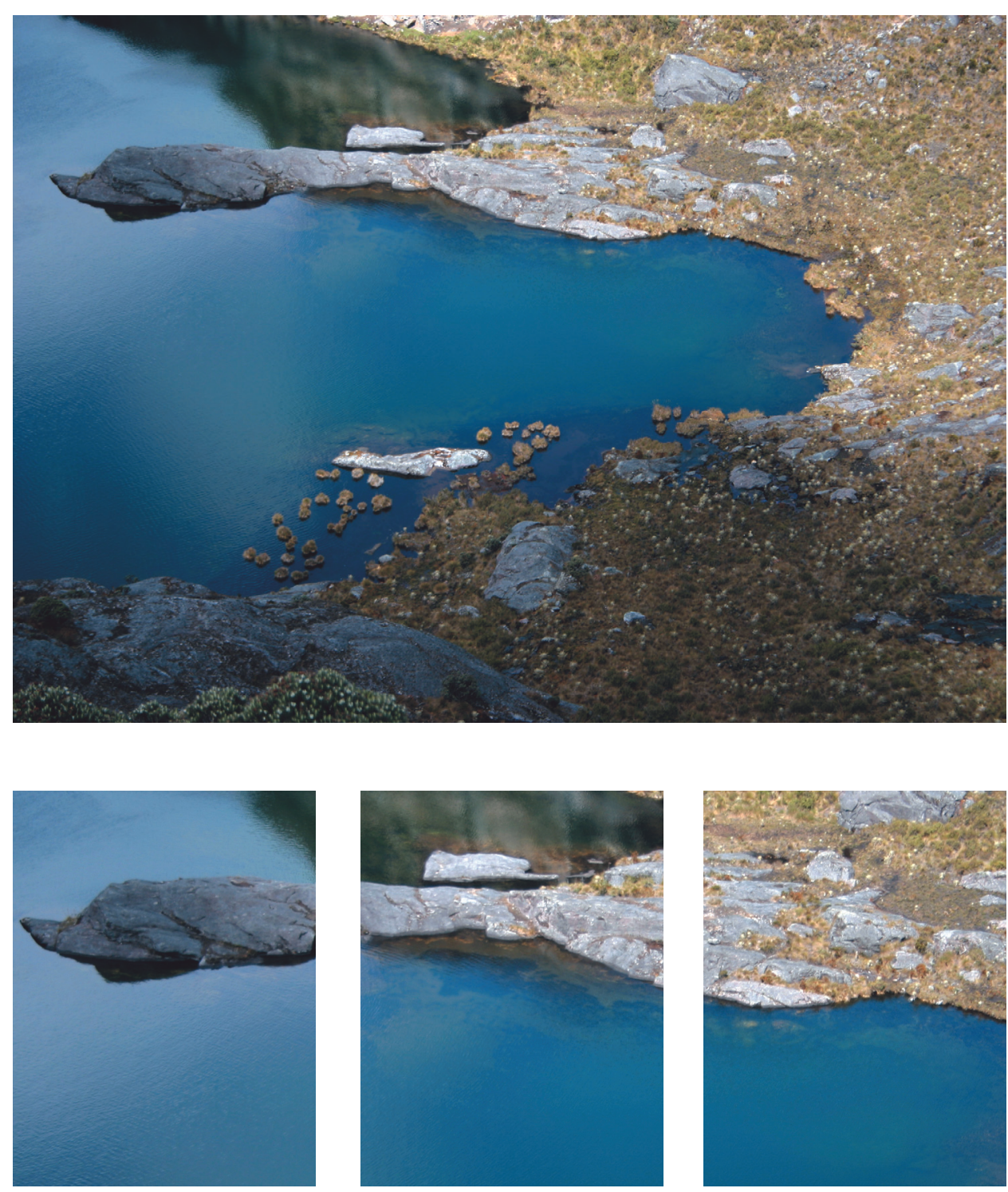

Península de la Laguna "La Pintada" 


\section{PARTICIPACIÓN CIUDADANA Y CONTROL SOCIAL: LOS SERVICIOS PÚBLICOS DOMICILIARIOS EN BUCARAMANGA, (COL)*}

Eva del Pilar Plata Sarmiento

\section{Introducción}

En su parte orgánica, la Constitución Política de 1991 consagra en el artículo 365, el derecho de participación ciudadana en materia de servicios públicos domiciliarios, con el objeto de asegurar la intervención de los usuarios en el ejercicio de la función de gestión y fiscalización sobre las distintas empresas prestadoras. La inclusión en la norma de normas de este derecho, teóricamente garantiza la efectiva prestación de dichos servicios a nivel nacional, toda vez que permite una comunicación fluida entre los distintos actores a fin de dar respuesta oportuna a los requerimientos ciudadanos.

La funcionalidad de los Comités de Desarrollo y Control Social, figura dispuesta legalmente para la organización de los usuarios de los servicios públicos domiciliarios, se encuentra relacionada en una proporción directa con el ejercicio y control del poder político que ejerza la ciudadanía, y con el conocimiento que se tenga de los mecanismos de participación.

* El artículo es producto del informe final del proyecto de investigación: Participación ciudadana en los Comités de Desarrollo de las Comunas 5, 6, y 7 de Bucaramanga. La investigación corresponde a la Línea Neoconstitucionalismo y Convivencia Pacífica, del Grupo de Investigación Neoconstitucionalismo y Derecho. Facultad de Derecho. Universidad Santo Tomás- Seccional Bucaramanga $(\mathrm{Col})$. 
El presente texto, a partir de las premisas anteriores y en el marco de la investigación 'Participación ciudadana en los Comités de Desarrollo y Control Social de las Comunas 5, 6 y 7 de Bucaramanga', estudia la relación existente entre participación ciudadana y funcionalidad de los Comités, determinando su incidencia en la gestión pública de los servicios públicos domiciliarios, indicador que ha de servir de instrumento para la elaboración de políticas públicas en el área y el planteamiento de futuras investigaciones.

\section{Demografía, situación socioeconómica y caracterización de Bucaramanga y sus Comunas 5, 6 y 7}

Bucaramanga (Colombia) se encuentra ubicada en el nororiente del país, es la capital de Santander, con un área de 165 kilómetros cuadrados donde habitan aproximadamente un millón de habitantes. En materia económica, la ciudad se distingue por su desarrollo en las industrias de calzado, la confección, la prestación de servicios de salud, finanzas y educación. El suelo urbano se encuentra dividido en 17 comunas $^{1}$ cada una de las cuales, desde el punto de vista territorial, agrupa un conjunto de barrios con servicios afines y complementarios en común, con características y vínculos socioeconómicos homogéneos (Plata, 2008, 84).

La comuna 5, denominada García Rovira, es un sector residencial cuya población para el año 2005 ascendía a 40.240 personas, lo cual representa el $7.89 \%$ del total de habitantes de Bucaramanga. Desde el punto de vista socio-económico es una zona de múltiples contrastes, en la que confluyen asentamientos subnormales caracterizados por las condiciones de pobreza junto con familias de ingresos suficientes para alcanzar una calidad de vida digna.

Los moradores del lugar presentan un nivel de formación académica bajo, el $35.7 \%$ ha cursado estudios completos de primaria, el $42.9 \%$ de secundaria y únicamente el $10.6 \%$ de pregrado o de niveles técnicos. En materia de cobertura de servicios públicos domiciliarios, el Plan de Desarrollo de Bucaramanga 2008-2011 cita las cifras del Censo 2005 realizado por el DANE (Departamento Administrativo Nacional de Estadística), señala que de un total de 7896 viviendas ocupadas: 189 no cuentan con el servicio de energía eléctrica, 367 con el de alcantarillado, 252 con el de acueducto, 584 con el de gas natural y 1.219 no tienen disponibilidad de teléfono fijo.

La comuna 6, denominada La Concordia, es un sector residencial con una amplia zona dedicada al comercio. Para el año 2005, en este lugar de la ciudad habitaban 26.903 personas, lo cual representa el 5.27\% del total de la población del municipio.

La comuna es entendida como una subdivisión administrativa menor, que corresponde a una zona urbana, basada en la proximidad y muchas veces reforzada por el antagonismo con el barrio vecino. Diccionario de la Real Academia Española. Vigésima Segunda Edición. ESPASA. España. 2001. Pág. 609 
Sus habitantes socio-económicamente se caracterizan por gozar de un nivel de vida digna, producto de los ingresos salariales que reportan.

En referencia al nivel de formación educativa, el 27.1\% de los residentes del lugar ha finalizado estudios de primaria, el $40.4 \%$ de bachillerato y el $24 \%$ de pregrado o de niveles técnicos. De un total de 2651 viviendas ocupadas, se reportan: 14 viviendas sin servicio de energía eléctrica, 46 sin alcantarillado, 42 sin acueducto, 173 sin gas natural y 256 sin teléfono fijo, se alcanza una cobertura promedio del $98 \%$.

La comuna 7, denominada La Ciudadela, es un sector residencial con pequeñas zonas comerciales, su población para el año 2005 ascendía a los 53.160 habitantes, representa el $10 . \%$ de la población. Desde el punto de vista socio-económico, los moradores de este lugar de la ciudad reportan importantes ingresos que les permitan mantener una calidad de vida digna y gozar de bienestar.

Del total de personas ubicadas en esta zona, el 28.6\% presenta título de primaria, el $40.7 \%$ de bachillerato y el $22 \%$ de estudios tecnológicos o profesionales. En referencia a la cobertura de servicios públicos domiciliarios, de un total de 12708 hogares: 45 viviendas no cuentan con el servicio de energía eléctrica, 65 con el de alcantarillado, 68 con el de acueducto, 153 con el de gas natural y 420 no disponen de teléfono fijo.

\section{Aproximaciones teóricas}

Gestión pública y participación ciudadana: acercamiento ciudadano hacia la elaboración de sus políticas.

La crisis del Estado de Bienestar y de la política burocrática de la administración pública, generó durante los años ochenta, un replanteamiento de su papel frente al modelo organizacional adoptado en lo público, para dar paso a un nuevo enfoque estadual sustentado en prácticas típicas del sector privado, enmarcadas en el concepto de 'revolución gerencial', con el que se busca “ofrecer mecanismos más eficientes para la entrega de bienes y servicios y al mismo tiempo incrementar los niveles desempeño (Pérez, 2000, 84).

La reforma ha impulsado un cambio en la concepción del papel del Estado, basándose en el fomento de demandas públicas desde la base, vía participación ciudadana y sistema político. Según Tommasini (1994), entre las razones que motivaron la reforma del Estado en América Latina se destacan: en primer lugar, la transformación del modelo económico imperante desde mediados de siglo XX, basado en un sistema de sustitución de importaciones y alta subsidiaridad estatal (proteccionismo), el cual entra en declive y expresan la crisis económica de la época y el crecimiento desmesurado del aparato estatal, hechos que incentivan un cambio de paradigma.

Un segundo hecho se refiere a la tendencia de revaloración de la sociedad civil, originada por la protección de los derechos humanos y la abolición del sistema socialista, marcada por un hecho trascendental: la caída del muro de Berlín. Y por 
último el acelerado proceso de nuevo conocimiento, o revolución tecnológica en palabras de Ohmae (2005).

Por tanto, el modelo emergente exigió al Estado cambiar su estrategia regulatoria para lograr la coordinación, eficacia y equidad en sus funciones. En este sentido, afirma que

"cuando un modelo económico cambia, consiguientemente sus políticas de desarrollo también, es inevitable que cambie el Estado, el derecho y su institucionalidad" (Tommasini, 1994).

Entre las políticas del nuevo modelo se destaca la liberalización de los mercados, flexibilización de los mismos, internacionalización de la economía, delegación de funciones de provisión pública a entes privados, descentralización y cambio tecnológico.

La nueva concepción estatal incentivó entonces un acercamiento mayor hacia la ciudadanía, sus necesidades y entorno. Es así como se crean nuevas herramientas de gestión pública enfocadas a tal fin mediante modelos gerenciales, la planeación estratégica y planeación participativa, entre otros, fueron las denominaciones más usadas.

En Colombia, la participación ciudadana es una de las herramientas previstas en el sistema normativo, con el propósito de integrar a los ciudadanos en el desarrollo de la gestión pública, en la elección de mandatarios, "en los procesos decisorios no electorales que incidirán significativamente en el rumbo de su vida" ${ }^{2} \mathrm{y}$, en la promoción de la satisfacción de los intereses colectivos; en otras palabras, la participación es el derecho de los ciudadanos a tomar parte en los asuntos públicos (Penagos, 1989, 277).

En el imaginario social se ha reforzado la equívoca idea de que el escenario político es el único en el cual los particulares tienen la oportunidad de intervenir, bien sea a través de la típica fórmula "elegir y ser elegidos", o mediante el uso de otros mecanismos como la revocatoria del mandato o la iniciativa legislativa.

La participación ciudadana, sin embargo, comprende toda

“(...) actividad individual o colectiva de la población dirigida a expresar y defender sus intereses, tomar parte en la administración de los asuntos comunes y en los centros de poder público, tanto en la toma de decisiones como en el control de la acción gubernamental..." (Pachón, 1997, 77),

lectura constitucional que deriva del preámbulo de la norma de normas, el cual expresa el poder soberano que inviste al pueblo, y de la consagración que en tal sentido realiza el mismo texto de la carta al señalar como un fin esencial del Estado, el

“facilitar la participación de todos en las decisiones que los afectan y en la

2 Corte Constitucional, sentencia C-180 de 14 de noviembre de 1994, Magistrado ponente: Hernando Herrera Vergara. 
vida económica, política, administrativa y cultural de la Nación".

El poder legislativo, de hecho, ha dispuesto distintas herramientas de participación, las cuales en ocasiones los ciudadanos no utilizan, no obstante que:

“(...). De la participación se obtienen beneficios sociales concretos: consecución de soluciones con el aporte colectivo; identificación entre los requerimientos de los ciudadanos y las posibilidades de servicio del Estado; funcionalidad de la gestión pública; mayor aceptación de la acción del Estado y, por consiguiente, de su legitimidad..." (Ibídem, 79-80).

En este sentido, Canto (2004) menciona las ventajas de la participación ciudadana en la función social del Estado, argumentando que ésta: [...] proporciona información sobre el ambiente social en el que se ejecutarán las actividades de desarrollo; revela de manera más eficiente las preferencias de los usuarios; genera aprendizajes sociales e innovación; fortalece a las instituciones locales; genera mayor credibilidad y legitimidad sobre las evaluaciones; contribuye a mejorar la eficiencia de las instituciones locales; genera formación de capital social; fortalece la competitividad sistémica de la región o localidad; contribuye a la formación y/o fortalecimiento de identidad local o regional.

La participación ciudadana, desde tal perspectiva, tiene un impacto positivo en el desarrollo de la gestión pública al promover una fluida relación y comunicación entre los prestadores del servicio público y los usuarios, situación que va a redundar, no sólo en una mejor prestación del servicio, sino también en la toma de decisiones acertadas que respondan a las demandas y prioridades de la comunidad, por parte de quienes suministran el servicio, para generar mayor bienestar social.

El ciudadano, incrustado en las organizaciones de carácter cívico y legal dispuestas para participar, se convierte así, en un actor clave, vocero de las necesidades que reporta el entorno del cual hace parte.

\section{Servicios públicos}

Las continuas transformaciones que caracterizaron al siglo $\mathrm{XX}$, debido a la crisis del Estado y al cambio de paradigma sobre la Administración Pública en los años 80, generaron un replanteamiento en punto a los mecanismos que éste puede utilizar para alcanzar los fines que se ha propuesto, y el surgimiento de nuevos modelos estaduales que propendían por el mejoramiento de la calidad de vida y la participación activa de los particulares en lo público a fin de alcanzar dichos objetivo.

La noción de servicio público, en este contexto, presentó varios cambios y se erigió como un medio para hacer efectivo el elemento social y humano que incorporaron varios países a la organización estatal, incluido el colombiano, al adoptar el modelo de Estado Social de Derecho. Así, el papel que debe cumplir aquél no se limita a 
garantizar un orden jurídico ${ }^{3}$, le corresponde además, asegurar el suministro ${ }^{4}$ de los servicios públicos, al responder las demandas ciudadanas y generar bienestar social.

Es tal la importancia de los servicios públicos
"que se llega a considerar que la principal actividad y preocupación de la administración debe consistir en asegurar cada día mejor [su] funcionamiento" (Rodríguez, 2005, 503).

Sobre el servicio público existen diferentes definiciones ilustrativas que esbozan los elementos que han de tenerse en cuenta para clasificar una actividad como tal, los cuales se revisten de importancia en un momento en el que "existe una tendencia a ubicar cada vez más actividades dentro de [esta] categoría”, situación resultado de la inclusión en los últimos tiempos de sectores económicos y acciones privadas en la realización de los fines estatales (Corte Constitucional, Sentencia T-520 de 2003).

Duguit $(1975,37)$, por ejemplo, define los servicios públicos como
“(...) toda actividad cuyo cumplimiento debe ser regulado, asegurado y fiscalizado por los gobernantes, por ser indispensable a la realización y al desenvolvimiento de la interindependencia social, y de tal naturaleza que no puede ser asegurado completamente más que por la intervención de la fuerza gobernante".

Según Marienhoff (citado por Orjuela, 1998, 80)

"por servicio público ha de entenderse toda actividad de la administración pública, o de los particulares o administrados, que tienda a satisfacer necesidades o intereses de carácter general cuya índole o gravitación, en el supuesto de actividades de los particulares o administrados, requiera el control de la autoridad estatal".

Para Bielsa (ibídem, 81), quien parte de una noción de servicio público amplio, para llegar a un concepto más delimitado, es

“toda acción o prestación realizada por la administración pública activa, directa o indirectamente, para la satisfacción concreta de necesidades colectivas, y asegurada esa acción o prestación por el poder de policía".

La ley misma también ha definido la noción de servicio público; el artículo 430 del Código Sustantivo del Trabajo en relación con la prohibición de la huelga, señala que se considera como tal

3 "El Estado Social de Derecho, se proyecta en la Constitución, en primer término, en la consagración del principio de igualdad y en su consecuencia obligada: los derechos sociales y económicos y en la prestación de los servicios públicos. En segundo término, a través de los derechos de participación de todos en las decisiones que los afectan y en la vida económica, política, administrativa y cultural de la nación, que se compendian en el principio democrático y gracias al cual se socializa el Estado y las diferentes instancias de poder dentro de la comunidad". Corte Constitucional, sentencia C-566 de 30 de noviembre de 1995, magistrado ponente: Eduardo Cifuentes Muñoz.

4 Dentro de las transformaciones que sufrió el concepto servicio público se encuentra la posibilidad de que la prestación de éste la realicen los particulares, por lo cual el deber del Estado es asegurar el suministro de los servicios públicos y no prestarlos directamente como en inicio lo sostuvo una parte de la doctrina. 
“(...) toda actividad organizada que tienda a satisfacer necesidades de interés general, en forma regular y continua, de acuerdo con un régimen jurídico especial, bien que se realice por el Estado directa o indirectamente o por personas privadas".

La Corte Suprema de Justicia en términos similares, indica que un servicio público es

“(...) toda actividad encaminada a satisfacer una necesidad de carácter general, en forma continua y obligatoria, según las ordenaciones de Derecho Público, bien sea que su prestación esté a cargo del Estado directamente, o de concesionarios o administradores delegados, o a cargo de simples personas privadas" 5 .

Se evidencia entonces, la adopción legal, jurisprudencial y doctrinal en Colombia de un concepto funcional en el cual

"el carácter de servicio público no depende de quién desarrolle la actividad, sino de las funciones sociales que ésta cumple" (Corte Constitucional, Sentencia T-520 de 2003).

\section{Servicios públicos domiciliarios}

La Constitución de 1991 en el artículo 367 establece una categoría especial de servicios públicos, los servicios públicos domiciliarios, sin definir ni determinar cuáles son,

"lo cual le corresponde a la ley, la que [además] debe establecer las competencias y responsabilidades correlativas, su cobertura, calidad y financiación, y el régimen tarifario respectivo" (Corte Constitucional, Sentencia C-585 de 1995).

El legislador, al cumplir tal mandato, aun cuando realizó un enunciado taxativo de cuáles deben tenerse por tales (artículo 14, numeral 21 de la Ley 142 de 1994), no precisó qué debe entenderse por servicio público domiciliario; siendo necesario postular una noción que permita comprenderlos.

La Corte Constitucional, por ejemplo, los ha definido como

"aquellos que se prestan a través del sistema de redes físicas o humanas con puntos terminales en las viviendas o sitios de trabajo de los usuarios y cumplen la finalidad específica de satisfacer las necesidades esenciales de las personas".

5 Sentencia de la Corte Suprema de Justicia, Sala Plena, Providencia de 18/VIII/70, M. P. Eustorgio Sarria. (Citado por Corte Constitucional, Sentencia T-520 de 2003).

6 Concepto reiterado en Sentencia T-734 de 2009, M. P. Jorge Iván Palacio Palacio. 
Asimismo, ha señalado las características que a su juicio resultan relevantes para determinar que un servicio público es domiciliario:

“a. El servicio público domiciliario -de conformidad con el artículo 365 de la Constitución-, puede ser prestado directamente o indirectamente por el Estado, por comunidades organizadas o por particulares, manteniendo éste la regulación, el control y la vigilancia de los servicios.

b. El servicio público domiciliario tiene una 'punto terminal' que son las viviendas o los sitios de trabajo de los usuarios, entendiendo por usuario 'la persona que usa ciertos servicios, es decir, quien disfruta el uso de cierta cosa'.

c. El servicio público domiciliario está destinado a satisfacer las necesidades básicas de las personas en circunstancias fácticas, es decir, en concreto"7.

Parámetros importantes, fundados en un criterio finalista, que han sido acogidos por un sector mayoritario de la doctrina, a partir del cual se puede definir servicio público domiciliario como una modalidad específica del servicio público que reciben las personas en su domicilio o lugar de trabajo para satisfacer las necesidades básicas de bienestar y salubridad.

\section{Participación y control social}

La participación directa de los usuarios de los servicios, como de la comunidad en general, está permitida por el Estado a través del control social, entendido como "la intervención de la comunidad como interlocutora válida, que exige el modelo de Estado colombiano", en procura de una gestión eficiente.

$7 \quad$ El criterio adoptado corresponde a la definición planteada en Sentencia T-578 de 1992, M. P. Alejandro Martínez Caballero.

8 "La noción del control social da vida a parte de los fundamentos del Estado Social de Derecho proclamado por nuestra Constitución Política. El modelo de Estado colombiano exige la vinculación directa de la ciudadanía al ejercicio de lo público en tanto que la asume no solamente como depositaria de sus intervenciones, sino también y muy especialmente como interlocutora válida y necesaria para garantizar una gestión efectiva. Por ello, el artículo 2 de la Constitución establece que entre los fines esenciales del Estado está 'facilitar la participación de todos en las decisiones que los afectan y en la vida económica, política, administrativa y cultural de la Nación’. Igualmente el 103 señala que 'El Estado contribuirá a la organización, promoción y capacitación de asociaciones profesionales, cívicas, sindicales, comunitarias, benéficas o de utilidad común no gubernamentales sin detrimento de su autonomía, con el objeto de que constituyan mecanismos democráticos de representación, en las diferentes instancias de participación, concertación, control y vigilancia de la gestión pública que se establezcan'. A estos principios responde el control social, pues se piensa como el ejercicio consciente, permanente y juicioso de ciudadanos y ciudadanas que se comprometen con el propósito de hacer seguimiento a la actividad del Estado y sus instituciones para que ellas cumplan con su sagrada misión y lo hagan desde la interlocución con quienes son sus usuarios y beneficiarios, no sus clientes porque al Estado no le corresponden los clientes". Tomado de: www.personeriabogota.gov.co 
La noción de control permite que la ciudadanía se vincule directamente en las diferentes instancias de participación, en el seguimiento a la actividad del Estado y sus instituciones para que cumplan la misión que se les ha encomendado, la cual no sólo se materializa en el cumplimiento meramente formal de las funciones que le han sido asignadas, sino en la realización efectiva de las tareas públicas y en la búsqueda permanente de la satisfacción de las necesidades generales de todos los habitantes (Corte Constitucional, Sentencia C-558 de 2001).

En el campo de los servicios públicos domiciliarios, en todos los municipios han de funcionar comités de desarrollo y control social, a razón de uno por cada servicio, integrados por un número de 50 a 200 usuarios activos o potenciales. Cada comité elige un vocal de control facultado para actuar como representante ante la respectiva empresa y las autoridades, con las siguientes funciones: Proponer a la empresa soluciones a las deficiencias; promover la concertación con la comunidad para la expansión y mejoramiento de los servicios; solicitar modificaciones a la estratificación; estudiar y formular recomendaciones sobre la asignación de subsidios y los criterios de reparto; solicitar al personero la imposición de multas hasta de diez salarios mínimos mensuales a la empresa prestadora por infracciones a las normas en perjuicio de los usuarios (Plata, 2008, 86).

\section{Desarrollo constitucional y legal}

La Constitución Política de Colombia de 1991 dedicó el capítulo 5 del Título XII a los servicios públicos, instituyendo un nuevo régimen en el tema, el cual propende por la prestación eficiente de los mismos, el mejoramiento de la calidad de vida de todos los habitantes del territorio nacional y la participación de la población usuaria y reserva para el Estado la regulación y vigilancia de dichos servicios. La norma, en lo que interesa a la presente investigación, consagra textualmente los siguientes principios:

- Es deber del Estado asegurar la prestación eficiente de los servicios públicos a todos los habitantes del territorio nacional.

- Los servicios públicos estarán sometidos al régimen jurídico que fije la ley, podrán ser prestados por el Estado, directa o indirectamente, por comunidades organizadas, o por particulares.

- El Estado mantendrá la regulación, el control y la vigilancia de dichos servicios.

- La ley fijará las competencias y responsabilidades relativas a la prestación de los servicios públicos domiciliarios, su cobertura, calidad y financiación, y el régimen tarifario que tendrá en cuenta además de los criterios de costos, los de solidaridad y redistribución de ingresos.

- La ley determinará los deberes y derechos de los usuarios, el régimen de su protección y sus formas de participación en la gestión y fiscalización de las empresas estatales que presten el servicio. Igualmente definirá la 
participación de los municipios o de sus representantes, en las entidades y empresas que les presten servicios públicos domiciliarios.

- Corresponde al Presidente de la República señalar, con sujeción a la ley, las políticas generales de administración y control de eficiencia de los servicios públicos domiciliarios y ejercer por medio de la Superintendencia de Servicios Públicos Domiciliarios, el control, la inspección y vigilancia de las entidades que los presten.

En este nuevo régimen de servicios públicos, como se observa, le fueron otorgados diferentes medios de inspección y regulación al Estado; es prioridad el interés social, la solución de necesidades básicas insatisfechas, la creación de subsidios para el pago de las tarifas de los servicios públicos domiciliarios de la población menos favorecida ${ }^{9}$, la participación ciudadana, la fiscalización y control de las empresas oficiales o privadas que prestan servicios públicos; y la vigilancia del Presidente de la República por medio de la Superintendencia de Servicios Públicos Domiciliarios cuya naturaleza, estructura y funciones fueron señaladas en la Ley 142 de $1994^{10}$.

Existen asimismo, en cuanto al control estatal se refiere, las Comisiones de Regulación, entidades eminentemente técnicas, creadas igualmente por la mentada disposición legal, cuyo propósito es, tal como su nombre lo indica, regular las actividades de los servicios públicos domiciliarios a fin de ampliar la cobertura y garantizar la calidad. En la actualidad existen tres (3): la Comisión de Regulación de Telecomunicaciones (CRT), la Comisión de Regulación de Energía y Gas (CREG) y la Comisión de Regulación de Agua Potable y Saneamiento Básico (CRA).

En cuanto al desarrollo legal de la materia, la Ley 142 de 1994, con sus correspondientes modificaciones, constituye el marco referencial en el tema de servicios públicos domiciliarios ${ }^{11}$, cuyo ámbito de aplicación se extiende también a las actividades complementarias de tales servicios y a otros servicios que sin ser domiciliarios la ley así lo determina.

Al respecto, la disposición legal bajo los lineamientos constitucionales reseñados se caracteriza por la defensa de los derechos de los usuarios y enfatiza en el ejercicio del control ciudadano sobre la prestación que de los servicios públicos realizan las

$9 \quad$ En el nuevo esquema el concepto de gratuidad quedó atrás, ya que los usuarios contribuyen al mantenimiento de las empresas a través del cargo fijo en aquellos casos contemplados, y el Estado asume una posición de garante en la prestación de los servicios, sin ejercer un monopolio sobre aquellos.

10 La Superintendencia de Servicios Públicos Domiciliarios es un organismo de carácter técnico cuyo fin es asegurar el control y vigilancia estatal de las empresas dedicadas a la prestación de servicios públicos domiciliarios

11 "Por su carácter de Ley especial la Ley 142 de 1994, en materia de servicios públicos domiciliarios debe aplicarse en forma preferente a otras disposiciones generales que hagan referencia a otras materias pero que podrían ser aplicables a otros servicios o a las actividades que realicen los prestadores de los mismos" (Atehortúa, 2003, 68). 
empresas, por medio de los Comités de Desarrollo y Control Social, figura jurídica que crea y desarrolla.

\section{Desarrollo de la investigación}

El objeto de la presente investigación fue preponderantemente analizado bajo la óptica del enfoque cuantitativo, se busca la posibilidad de generalizar resultados y hallazgos de la misma en términos de magnitudes, mediciones y categorías. Sin embargo, debido a la particularidad del tema a tratar y su configuración social se tuvieron en cuenta elementos propios del enfoque cualitativo, especialmente busca sustraer percepciones, información más amplia y cualidades del proceso objeto de estudio así como de los directamente implicados.

En este marco, el presente trabajo asumió un alcance descriptivo, según el cual se especificaron características propias de la participación de la ciudadanía de las comunas 5, 6 y 7 de Bucaramanga en la prestación de los servicios públicos domiciliarios y más específicamente el papel que ejercen los Comités de Desarrollo y Control Social.

La investigación partió de la base de la consolidación del Estado del arte sobre la materia, especialmente aquellos trabajos producto de investigación dirigidos a la medición de la participación ciudadana en el sector de los servicios públicos a nivel local o nacional, sobre el control social ejercido a entidades o empresas que prestan servicios públicos, sobre la participación de la comunidad en organizaciones que promuevan la defensa de los derechos de usuarios de servicios públicos y ejerzan vigilancia sobre las empresas que los prestan, entre ellas, los Comités de Desarrollo y Control Social. Asimismo se abordó los antecedentes normativos y la regulación vigente así como los parámetros establecidos en el tema por la Corte Constitucional, el Consejo de Estado y la Superintendencia de Servicios Públicos Domiciliarios, en relación con los pronunciamientos sobre temas como: participación ciudadana y control social, definición de servicios públicos domiciliarios, derechos de los usuarios de servicios Públicos Domiciliarios, creación, inscripción y demás generalidades de los Comités de Desarrollo y Control Social. Todo esto con la finalidad de entender el funcionamiento, dinámica y razón de ser de la participación ciudadana ejercida por medio de los Comités de Desarrollo y Control Social. Todo ello se relacionó con los aportes teóricos de trabajos relativos al tópico de estudio. Igualmente se realizó una caracterización demográfica, geográfica y socioeconómica de las comunas 5, 6 y 7 de Bucaramanga, así como del funcionamiento y operatividad de cada uno de los Comités de servicios públicos, con cada uno de los elementos anteriores se procedió a aplicar cada uno de los instrumentos idóneos de recolección de datos seleccionados. 


\section{Participantes}

Los participantes directos de la investigación fueron los ciudadanos que conforman las comunas 5, 6 y 7 de la ciudad de Bucaramanga. Asimismo, los integrantes de los Comités de Desarrollo y Control Social. Los primeros se seleccionaron de acuerdo al censo 2005 del Departamento Administrativo Nacional de Estadística [DANE], según el cual las tres comunas objeto de estudio habitan 120.303 habitantes, para los cuales de acuerdo a un muestreo aleatorio simple con asignación proporcional se aplicaron 783 encuestas con un error máximo aceptable de 5\%. De tal muestra seleccionada se aplicó el instrumento en los sectores de las comunas 5, 6 y 7 por medio de un sondeo casa por casa proporcional al tamaño de cada conjunto residencial y su importancia en la asignación muestral.

Cuadro 1. Encuestas aplicadas por sectores

\begin{tabular}{|c|c|c|c|c|}
\hline COMUNA & POBLACIÓN & $\%$ & MUESTRA & $\begin{array}{c}\text { MUESTRA } \\
\text { APROXIMADA }\end{array}$ \\
\hline García Rovira & 40.240 & $33 \%$ & 262,2 & 262 \\
\hline Concordia & 26.903 & $22 \%$ & 175,3 & 175 \\
\hline Ciudadela & 53.160 & $44 \%$ & 346,4 & 346 \\
\hline Total & 120.303 & $100 \%$ & 784 & 783 \\
\hline
\end{tabular}

Fuente: Elaboración propia

Los miembros de los Comités de Control Social fueron el segundo tipo de participantes, es de destacar que en la Comuna 5, no fue posible contactar a los miembros de dicho comité, únicamente se contó con la entrevista de dos vocales. Asimismo, en la Comuna 6 respecto a los miembros de Comités de desarrollo y control social, no obstante desarrollar todas las acciones necesarias para contactarlos, no fue posible entrevistar a ningún vocal, por motivos similares a los señalados en la Comuna 5.

\section{Instrumentos}

Dos fueron los instrumentos aplicados como estrategia de recolección de datos, los cuales conformaron la evidencia necesaria para describir la dinámica objeto de estudio. El primero se configuró por medio de una encuesta aplicada directamente a los usuarios de los servicios públicos domiciliarios del espacio seleccionado, la cual indagaba acerca de configuración socioeconómica del encuestado, conocimiento de organizaciones comunitarias, utilización de servicios públicos domiciliarios, los derechos y deberes de los usuarios de servicios públicos, los problemas más 
frecuentes y los canales de comunicación de los mismos, información acerca de las empresas de servicios públicos y su accesibilidad y, por último, cuestionamientos relativos al conocimiento y participación en los mecanismos de toma de decisiones ciudadanas, por medio de los Comités de desarrollo y control social.

El segundo fue la entrevista estructurada, a partir de un cuestionario de preguntas prediseñadas dirigidas a los miembros de los Comités de Control Social alusivas a indagar el conocimiento acerca de los mecanismos de participación, su afiliación a alguno de ellos, conocimiento acerca del funcionamiento y operatividad de los comités y su utilización, calidad y oportunidad de la información y formación de programas que fomenten el mejoramiento de los servicios públicos.

La información anterior, sirvió para determinar aspectos de funcionalidad de los comités, ejercicio de la función de vigilancia y control a su cargo, desempeño en la zona en que se han constituido, permanencia, apertura y utilización de espacios en forma periódica para información de gestión adelantada por el vocal y miembros del comité, sostenibilidad del comité, grado de conocimiento de las herramientas de participación ciudadana, defensa del usuario, caracterización de quejas, reclamos o peticiones promovidas, participación ciudadana para la mejora de los servicios y promoción programas ambientales, entre otros.

\section{Resultados}

\section{Nivel 1. Identificación de los requerimientos ciudadanos}

Los resultados obtenidos indican que el $100 \%$ de las personas que habitan en las comunas objeto de estudio, identifican los servicios públicos domiciliarios y son usuarios de los mismos, condición que les confiere una serie de derechos que concretan principalmente en recibir los servicios públicos junto con la facturación que el consumo de los mismo genere, y obtener una respuesta oportuna a los reclamos que formulen y a los daños que reporten.

No obstante lo anterior, la totalidad de la población no conoce las actividades realizadas por las empresas prestadoras, únicamente la mitad de los encuestados referencian datos relacionados con su accionar, información que obtienen a través de los medios de comunicación (radio, prensa y televisión) y de la factura.

Los encuestados señalan igualmente como propios una serie de deberes, a saber: cancelar oportunamente las facturas, permitir la lectura de los medidores (contadores) a los empleados de las empresas de los servicios públicos domiciliarios, ahorrar los servicios públicos, vigilar las empresas prestadoras de los servicios, y cuidar los contadores. 
Nivel 2. Reconocimiento de los mecanismos por parte de los ciudadanos para ejercer el control social en los servicios públicos domiciliarios.

Los ciudadanos, ante el interrogante de si conocen comités o asociaciones comunitarias, evidenciaron una ausencia de reconocimiento en cuanto a las distintas formas de organización ciudadana, dado el alto porcentaje de respuestas negativas. Quienes respondieron afirmativamente, identifican como tales a las acciones comunales, los grupos religiosos, los comités deportivos y los grupos juveniles.

En punto a la posibilidad de organizarse en un ente que represente su comunidad en materia de servicios públicos domiciliarios, un porcentaje mayoritario de los encuestados no la vislumbra, lo cual refleja un bajo nivel de asociatividad; el grupo minoritario que si ha contemplado la idea, considera que podrían asociarse a través de la Junta de Acción Comunal, una Veeduría Ciudadana y, Comités de Consumo de los Servicios.

Las personas que conformaron la muestra asimismo manifestaron mayoritariamente no saber qué es un comité de desarrollo y control social; quienes se pronunciaron en sentido contrario, indicaron que la función de dicho comité consiste en asesorar a los usuarios, vigilar a los usuarios, fiscalizar a la empresas, ayudar a los usuarios a pagar las facturas de los servicios públicos domiciliarios.

Situación en conjunto que redunda en la imposibilidad de determinar por parte de los encuestados si existen en su barrio Comités de desarrollo y control social de los servicios públicos, y en la ausencia de participación a nivel comunal, efecto último que es atribuido expresamente a la incertidumbre respecto de cómo hacer parte y a la falta de información.

\section{Nivel 3. Consecución de soluciones}

Frente a las distintas problemáticas referentes a la prestación de los servicios públicos domiciliarios, las cuales fueron evidenciadas en el nivel I, los usuarios encuestados para solucionarla, optan por presentan una petición respetuosa a la empresa prestadora, comunicarse con ella, o por acudir a la Superintendencia de Servicios Públicos Domiciliarios.

\section{Conclusiones y Recomendaciones}

Las empresas prestadoras de servicios públicos domiciliaros, en el marco del Estado Social de Derecho, no pueden limitar su accionar a satisfacer las necesidades básicas de bienestar y salubridad propias del sector en el cual desarrollan su objeto, adicionalmente deben propender por una prestación eficiente, para buscar el mejoramiento de la calidad, y por ampliar la cobertura, llegar incluso a adoptar sistemas que compensen la incapacidad de pago de aquellos usuarios que no poseen 
los recursos suficientes para acceder a los mismos, cuestión a la cual no es ajena la legislación colombiana.

La Ley 142 de 1994, en consonancia con lo expuesto y en desarrollo del mandato incorporado en el artículo 369 de la Constitución Política, creó los Comités de Desarrollo y Control Social de los Servicios Públicos Domiciliarios como el medio idóneo para que los usuarios puedan ejercer un control social tendiente a hacer de lo enunciado una realidad.

La participación ciudadana, en este contexto, asume un papel fundamental en la medida en que contribuye a generar canales de comunicación que permiten a quien suministra el servicio realizar un balance de su gestión acorde a la realidad, para a partir de ello adoptar las decisiones necesarias a fin de responder acertadamente a las demandas y prioridades de la comunidad; al tiempo que ayuda al cumplimiento de la función de regulación y vigilancia radicada en cabeza del Estado, en el momento en que se informa a los organismos creados para tal propósito los resultados de la fiscalización realizada.

En Bucaramanga (Col.), ciudad en la cual los servicios públicos domiciliarios alcanzan índice de cobertura total (aproximadamente 99\% conforme con las cifras presentadas por el DANE en 2005), la investigación realizada evidencia una ausencia de participación de los usuarios en el control de los mismos, lo cual tiene como necesario efecto, conforme a lo señalado anteriormente, una baja incidencia en la gestión pública realizada por las empresas prestadoras y por el Estado.

Aunque los ciudadanos reconocen como propios una serie derechos derivados de la calidad de usuario, los cuales circunscriben a recibir el servicio y a obtener una respuesta a las reclamaciones presentadas, su campo de acción se encuentra limitado

"Al ser la participación activa de los ciudadanos la que promueve de forma efectiva el cumplimiento de los derechos fundamentales y colectivos" (Fernández \& Herreño, 2007, 65),

las pocas acciones tendientes a la consecución de soluciones a los requerimientos planteados por la colectividad no presenta mayor impacto.

De hecho, si bien los usuarios reconocen y señalan la existencia de diversas problemáticas en cuanto a la prestación y a la calidad, cuando actúan para buscar una solución se limitan a presentar peticiones respetuosas por individual o simplemente a comunicarse con la empresa, convirtiéndose en actores aislados.

Tal situación tiene origen en la falta de información y el desconocimiento mismo de la figura en torno a la que pueden agruparse para ejercer control social, tal como expresamente lo señalan los mismos encuestados; sin embargo, el estudio evidenció otras causas como: el desinterés por el tema, la posibilidad de participar únicamente en actividades breves o temporales, y el bajo nivel de asociatividad de algunos sectores, es decir, la poca disposición para establecer relaciones con otras personas a fin de trabajar mancomunadamente en pro de un(os) tema(s) determinado(s). 
La inexistencia y la falta de funcionalidad de los Comités de Desarrollo y Control Social de los Servicios Públicos Domiciliarios, observada al momento de aplicar las encuestas y realizar entrevistas a los vocales de control, precisamente no es más que la necesaria consecuencia de no conocer las actividades que desarrollan las empresas prestadoras, ni qué es y cómo opera un Comité de Desarrollo y Control Social, aunado a no vislumbrar la posibilidad de asociarse en un ente que represente a la comunidad en materia de servicios públicos.

Para superar tales falencias, a título de recomendación, se propone la divulgación por parte de las empresas prestadoras de las actividades que desarrollen acudiendo a los medios de comunicación y a la factura, sugerencia que también es predicable de los Comités de Desarrollo y Control Social existentes.

La Superintendencia de Servicios Públicos, en la misma línea, deberá desarrollar capacitaciones de fácil acceso tendientes a la promoción y conformación de los mentados Comités en aquellos lugares en los que éstos todavía no sean una realidad, labor que podrá desarrollar en asocio con la Alcaldía Municipal la cual deberá promover otras formas de organización ciudadana que estimulen la participación.

La labor de acompañamiento deberá prolongarse en el tiempo en aspectos como técnicas de planeación y actualización jurídica, dotar de los elementos necesarios a los vocales de control para llevar a cabo las funciones que el artículo 64 de la Ley 142 les ha encomendado.

\section{REFERENCIAS}

Amar, J. \& Echeverría, J. (2006). Participación comunitaria para el control ciudadano en los servicios públicos domiciliarios en el distrito de Barranquilla, en: Revista de Derecho, No 25. Universidad del Norte. Barranquilla - Colombia.

Atehortúa, C. (2003). Servicios Públicos Domiciliarios. Legislación y Jurisprudencia. Primera edición. Bogotá Colombia.

Caballero, G. \& Anzola, M. (1995). Teoría constitucional. Editorial Temis. S. A. Santafé de Bogotá - Colombia.

Canto, M. (2004). Participación ciudadana y reforma del Estado: ¿El gobierno hace a la participación o la participación hace al gobierno? IX Congreso Internacional del CLAD sobre la Reforma del Estado y de la Administración Pública, Madrid, España, 2 -5 Nov. 2004 Documento Libre. Recuperado de http://www.iij.derecho. ucr.ac.cr/archivos/ documentacion/inv\%20otras\%20entidades/CLAD/CLAD $\% 20$ IX/documentos/canto.pdf

Diccionario de la Real Academia Española. Vigésima Segunda Edición. ESPASA. España. 2001.

Duguit, L. (1975). Las transformaciones del derecho: público y privado. Heliasta. Buenos Aires - Argentina. 
Fernández, L. \& Herreño, D. (2007). Caracterización de la gobernabilidad en Santander, Colombia, en: Revista Reflexión Política $N^{\circ} 18$. Universidad Autónoma de Bucaramanga. Bucaramanga - Colombia.

Méndez, C. (2007). Metodología. Diseño y desarrollo del proceso de investigación. Cuarta edición. Editorial: Limusa Noriega Editores. México.

Ohmae, K. (2005). El próximo escenario global: desafíos y oportunidades en un mundo sin fronteras. Granica: Barcelona.

Olano, H. (2004). Preguntas y respuestas de derecho constitucional colombiano y teoría general del Estado. Segunda edición. Ediciones Doctrina y Ley. Bogotá Colombia.

Orjuela, C. (1998). La noción de servicio público esencial y sus efectos en el derecho del trabajo, en: El Derecho del trabajo y la seguridad social. Colegio de Abogados del Trabajo y Legis Editores. Bogotá - Colombia.

Pachón, C. (1997). Participación ciudadana y comunitaria. 2a . Edición. Ediciones Jurídicas Gustavo Ibáñez. Bogotá Colombia.

Penagos, G. (1989). Curso de derecho administrativo. Tomo II. 2a . Edición. Ediciones Librería del profesional. Bogotá - Colombia.

Pérez, Ignacio. (2000). La inversión en recursos humanos de excelencia y la nueva gerencia pública, en: Revista Centroamericana de Administración Pública, No 38-39. San José - Costa Rica.

Plata, E. (2008). Participación ciudadana y Control social: Los servicios públicos domiciliarios en Bucaramanga (Colombia), en: Revista IUSTITIA, No 6. Universidad Santo Tomás. Bucaramanga - Colombia.

Rodríguez, L. (2005). Derecho Administrativo. General y Colombiano. Decimocuarta edición. Editorial Temis. Bogotá - Colombia.

Sáchica, L. (1996). Nuevo constitucionalismo colombiano. Duodécima edición.1996. Editorial Temis S. A. Bogotá - Colombia.

Superintendencia de Servicios Públicos Domiciliarios. (Editor). (2004). Participación Ciudadana de Servicios Públicos Domiciliarios. Manual de Comités de Desarrollo y Control Social. Bogotá - Colombia.

Tommasini, L. (1994). La Reforma del Estado y las políticas públicas. Universidad de Chile. Centro de Análisis de Políticas. Andros Nataniel. Santiago, Chile.

Vidal, J; Díaz, V. \& Rodríguez, G. (2005). Temas de Derecho Administrativo Contemporáneo. Universidad del Rosario. Bogotá. Colombia.

Younes, D. (2004). Curso de derecho administrativo. Séptima edición. Editorial Temis, S. A. Bogotá - Colombia.

\section{Jurisprudencia}

Corte Constitucional Sentencia C-180 de 14 de noviembre de 1994, Magistrado ponente: Hernando Herrera Vergara. 
, Sentencia C-585 de 07 de diciembre de 1995, Magistrado ponente: Hernando Herrera Vergara.

- Sentencia C-242 de 20 de mayo de 1997, Magistrado ponente: Hernando Herrera Vergara.

Eduardo Cifuentes Muñoz. , Sentencia C-242 de 28 de mayo de 1997, Magistrado ponente: Antonio Barrera Carbonell.

Sentencia T-001 de 16 de enero de 1998, Magistrado ponente: , Sentencia C-444 de 26 de agosto de 1998, Magistrado ponente: Hernando Herrera Vergara.

Araujo Renteria. , sentencia C-558 de 31 de mayo de 2001, Magistrado ponente: Jaime Araujo Rentería.

, sentencia T-019 de 23 de enero de 2002, Magistrado ponente: Jaime

Corte Constitucional, sentencia T-447 de 30 de mayo de 2003, Magistrado ponente: Rodrigo Escobar Gil.

Escobar Gil. , sentencia T-520 de 26 de junio de 2003, Magistrado ponente: Rodrigo , sentencia T-1205 de 02 de diciembre de 2004, Magistrado ponente: Marco Gerardo Monroy Cabra.

Rodrigo Escobar Gil. , sentencia C -075 de 08 de febrero de 2006, Magistrado ponente: , sentencia T-734 de 15 de octubre de 2009, Magistrado ponente: Jorge Iván Palacio Palacio.

\section{Sitios Web}

Centro Latinoaméricano para el Desarrollo rural. RIMISP. http:/www. rimisp. cl/seccion.php?seccion=618 Consultado: Septiembre 20 de 2008.

Informe de la gestión de la Superintendencia de Servicios Públicos Domiciliarios. Vigencia 2007. www.superservicios.gov.co/sitesspd/ publicaciones.jps Consultado: Septiembre 20 de 2008.

Alcaldia de Bucaramanga. www.bucaramanga.gov.co

Personería de Bogotá. www.personeriabogota.gov.co 